\title{
Material Properties of 3D Printed Parts: Challenges in Design and Analysis
}

\author{
Madhukar Somireddy \\ Department of Mechanical Engineering \\ York University, Toronto, Canada
}

\author{
Aleksander Czekanski \\ Department of Mechanical Engineering \\ York University, Toronto, Canada
}

\begin{abstract}
The present paper addresses the challenges in design and analysis of 3D printed structures with anisotropic material properties. In recent years the additive manufacturing technologies have been used in most of the industries because of their freedom in fabrication of any complex geometry part. However, the final properties of the 3D printed parts are anisotropic though the material used is isotropic. Further, the properties of printed part are not homogeneous. This change in the properties is due to process parameters and build orientation of the parts. Therefore, this calls special attention of the designer for effective design and analysis of such parts. In this paper, design consideration of the parts for 3D printing and also, their structural analysis for effective design of parts for 3D printing are discussed. Further, different steps involved from design to 3D printing of a product are discussed.
\end{abstract}

Keywords-3D printing; Material properties; Design; Analysis;

\section{INTRODUCTION}

Additive manufacturing, also known 3D printing, fabricates 3D part by deposition of material layer upon layer. This layer by layer fabrication technology can build a part of any kind of geometry. It means that the technology facilitates more freedom for designing of any intricate shape of parts. The ASTM [1] classifications of AM processes based on process methodology are binder jetting, direct energy deposition, material extrusion, material jetting, powder bed fusion and sheet lamination and photo-polymerization. The other main benefits are rapid prototyping, fabrication of product with minimum part count, better structural integrity of product, no special tooling, flexibility for fabrication of wide range of products, no need of skilled operator, eliminates different sizes of stock materials and minimal or no wastage of processing materials. Furthermore, the AM techniques allow tailoring the material properties of the part by printing desired mesostructure during material deposition and also building functional graded parts much easier. The design of part for $3 \mathrm{D}$ printing is not same as traditional design for machining methods and the design rule for additive manufacturing is discussed by [2]. The material properties of the printed parts are differing form their initial properties and the effect of anisotropic material properties on structural behavior of the printed parts was addressed in [3]. Effect of process parameters on the material microstructure and design principle for additive manufacturing a part is available in [4]. The design of a part for 3D printing involves much more than just fabricating a three dimensional part. It involves design of microstructure, selection of printing direction, process parameters and build orientation. This paper addresses the effect of anisotropic material properties on the design of a part. Also, challenges in design and analysis of part for $3 \mathrm{D}$ printing are discussed. Then the different stages and the factors that need to be considered during design stage of a part for additive manufacturing process are presented.

\section{CHALLENGES IN DESIGN AND ANALYSIS}

The 3D printing of any complex geometry allows the designer for designing of parts of size microstructural (micrometers) to structural level (meter level). At the same time the designer needs to pay attention to the fabrication process and its process parameters while designing the part for $3 \mathrm{D}$ printing. The physical phenomena that take place during the layer upon deposition of the material have strong impact on the quality of the printed parts that includes mechanical properties. This is due to printing the microstructure of the part during layer upon layer material deposition and the mesostructure is mainly governed by the process parameters. This introduces the anisotropy in the material properties even though the isotropic material used for printing. Also, the degree of anisotropy depends on the type of AM process employed for printing the part. Furthermore, the material of the printed part is not homogeneous in different sections of the part. This inconsistency in the material properties of the printed part is major challenge to designer to specify the final material properties of the part. To account this change in the material behavior, the parts have to be designed not just based on geometrical analysis but also based on the phenomena that is taking places during printing. Therefore, it is essential to consider type of AM process and its process parameters while designing the parts for $3 \mathrm{D}$ printing.

The limitations of AM processes are range of materials for printing, dimensional accuracy, build time, support structures, surface finish, mass production, post processing and consistency in the material properties. These are mainly governed by the type of AM process and its process parameters. Also, the build orientation of the parts influences the build time, quality of the part and material properties. So far, the design of parts for printing is based on geometrical analysis, reduction in build time and cost, minimal support structures and for better surface finish. The anisotropy in the material properties of the printed part via different AM 


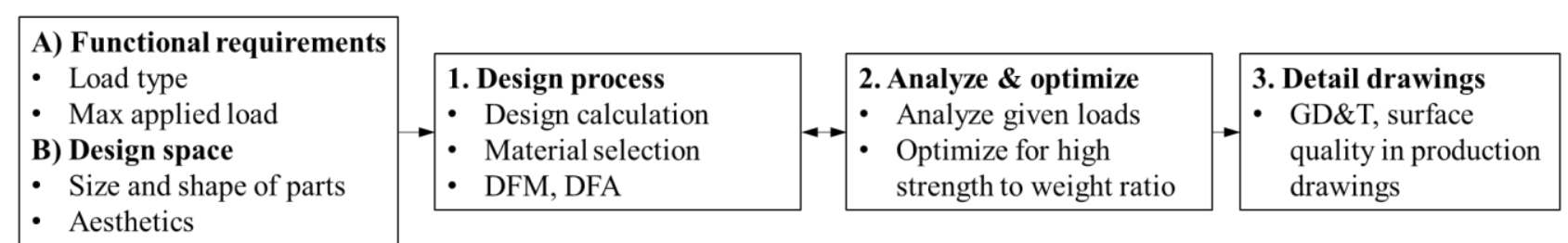

Figure 1. Stages in the product design, development and fabrication

processes was addressed [5]. Effect of the build orientation of the part and process parameters on the mechanical properties of the printed part via FDM was discussed in [6]. The anisotropic material behavior of printed parts via SLA [7]. The variation in the material properties of the printed part via SLM are discussed in [8]. Therefore, while designing a part for 3D printing the designer need to consider the influence of AM process and its process parameters on the final printed part. Further, the limitation of the process and influence of build orientation on quality and performance of the part are to be considered while designing. It means that for effective design and analysis of parts for 3D printing the designer need to have idea about the relationship between process-structure-propertyperformance.

The parts subject to certain loads are designed and analyzed for a selected material to get the its geometrical parameters. It means the geometrical features (size, shape) of the part derived from design and analysis calculations for desired strength to sustain the applied load on it. Different stages involved in the design and development of a product for traditional machining methods are shown in Fig. 1. The main inputs for the product design are its functional requirements and design space. The functional requirements of product include its purpose, load type and maximum applied load. Then the design space defines the maximum allowed size and shape of the product. Then the geometry of the parts of the product are designed for the applied load and selected material. This involves the design calculation that the stresses of the parts produced due to applied load lower than the strength of the selected material. Simultaneously during design stage, the design for manufacturability (DFM) and design for assembly (DFA) are considered for fabrication and assembly and disassembly of the parts of the product. Then in the next stage, the product is analyzed and optimized for higher strength to weight ratio. Finally, the detail drawing of the optimized parts are produced with the tolerances, surface quality requirements and suggestions of machining methods for fabrication of the parts. For instance, a part is to be designed for holding two shafts and subjects to load (P). The Fig. 2 explains different stages involved in design and analysis of the part.

The design of a part for 3D printing could not follow the same stages, because the challenges in designing and their limitations of AM processes as mentioned earlier. Anisotropy and inconsistent material properties of printed part are the main challenges during the design. Let us consider a $3 \mathrm{D}$ printed $\mathrm{L}$ bracket via FDM process. The bracket has two plates; horizontal and vertical plate and a cylindrical section to each of them. The horizontal plate is oriented on the substrate of the printer and the orientation of the vertical plate is perpendicular to the horizontal. As discussed earlier that the properties of the printed parts depend on process parameters and it build orientation. The material properties and behavior of the two plate would not be same because of their build orientation. The behavior of printed horizontal plate would be orthotropic and vertical is transverse isotropic. The $3 \mathrm{D}$ printing of $\mathrm{L}$ bracket with different build orientations is shown in Fig. 3 and their influence is presented in Table I. The other process parameters of FDM such as layer thickness, printing direction, percentage of infill, infill pattern, air gap between the fibers, temperature of build area and printing speed influence the mechanical properties of the printed part. Because of change in the material properties, the initial properties used in design calculations of a part for 3D printing do not provide reliable design output. That includes the geometrical feature of the part and expected material behavior of the part for applied loads. Besides that, results of analysis and optimization of the part based on initial properties are not correct. Because of such challenges, the design of a product for additive manufacturing (DFAM) is much more than the traditional design process for machining methods. The DFAM requires the designer to take care of type of AM process, material properties, build orientation, support structures, build time, cost and quality of the parts.
Input

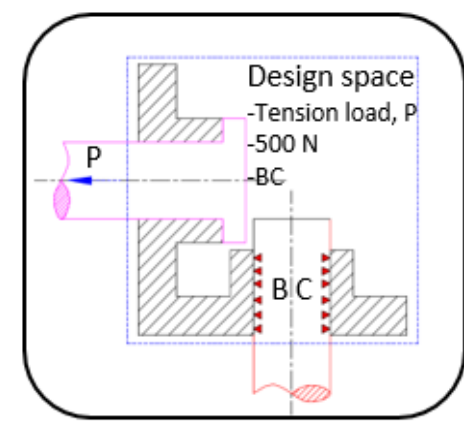

1. Design Process

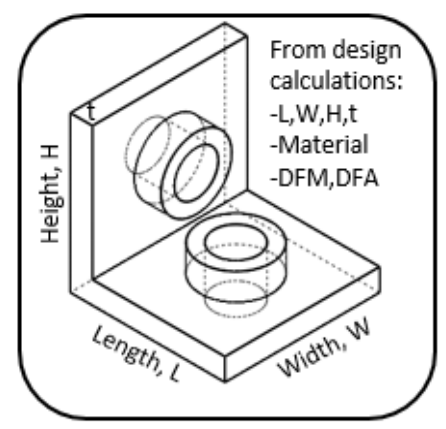

2. Analyze \& optimize

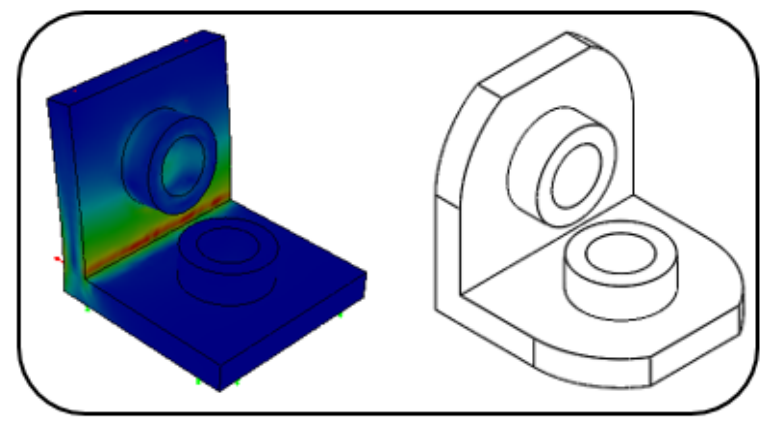

Figure 2. Stages in design and analysis of a part. 


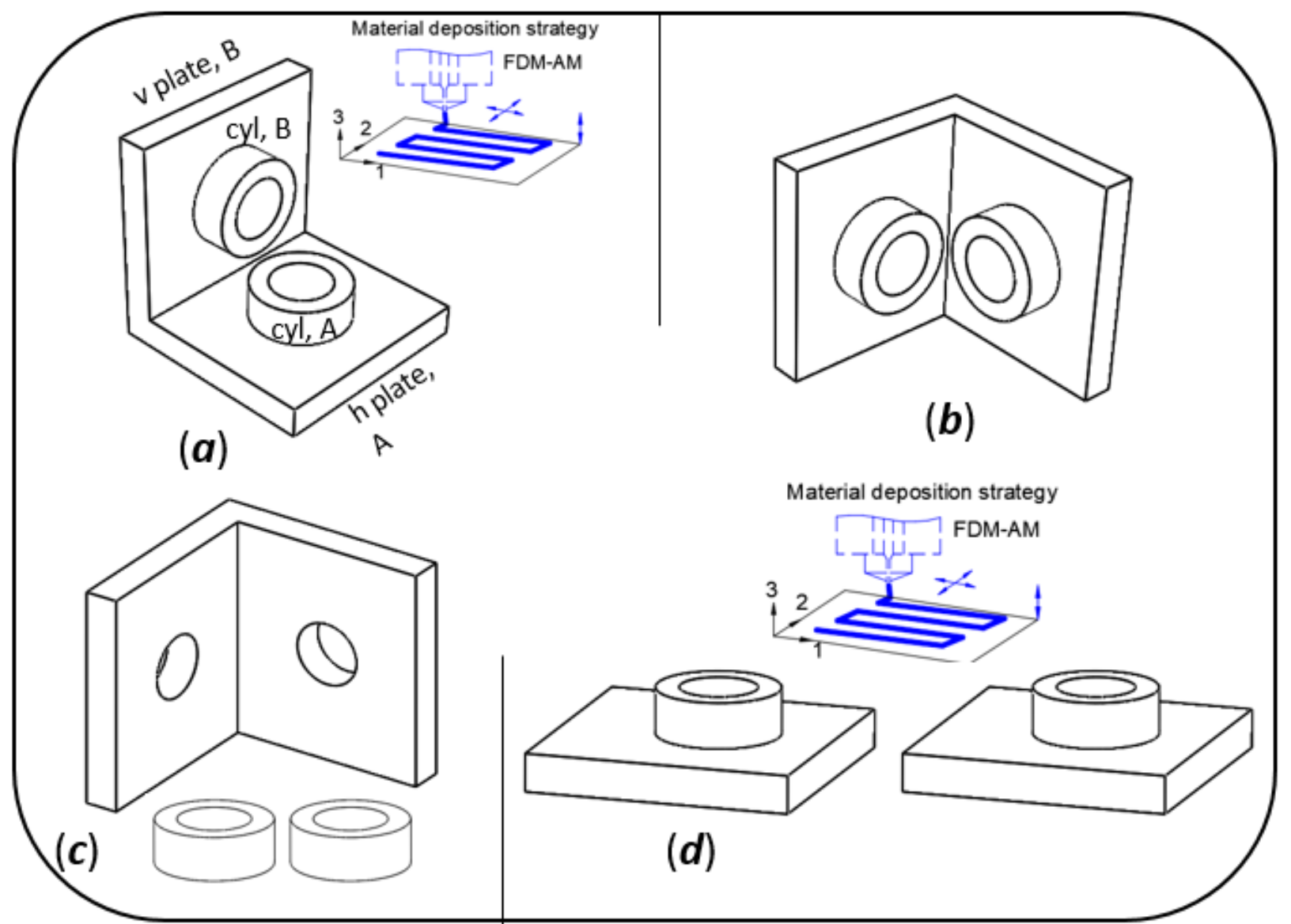

Figure 3. Different build orientations of the L bracket for 3D printing.

TABLE I. INFLUENCE OF BUILD ORIENTATION OF A PART IN 3D PRINTING.

\begin{tabular}{|l|c|c|c|c|}
\hline & $\mathbf{3}(\mathbf{a})$ & $\mathbf{3}(\mathbf{b})$ & $\mathbf{3}(\mathbf{c})$ & $\mathbf{3}(\mathbf{d})$ \\
\hline Properties of A,B & $\neq$ & $\neq$ & $=$ & $=$ \\
\hline Homogeneity in A, B & No & No & Yes & Yes \\
\hline Support structures & Cyl B & Cyl A, B & None & None \\
\hline Build time & Higher & Highest & Lower & Lower \\
\hline Assembly & None & None & Yes & Yes \\
\hline Structural integrity & Strong & Strong & Weak & Weak \\
\hline Post processing & Yes & Yes & No & No \\
\hline
\end{tabular}

A modified design process and the special design tools that consider the flow of information between the processstructure-property-performance could help the designer for effective design and analysis of the parts for 3D printing. The modified design process and steps involved during design of part for 3D printing is presented in Table II. The designer tools for 3D printing guides the designer based on the type of $\mathrm{AM}$ process and its process parameters for fabricating the parts. More importantly material data of the final printed parts based on the process parameters and build orientation for effective design and analysis of parts for 3D printing.
TABLE II. STEPS IN DESIGN OF A PART FOR 3D PRINTING.

\begin{tabular}{|c|c|c|}
\hline \multicolumn{3}{|c|}{ 1. Inputs; functional requirements, design space of product } \\
\hline \multicolumn{3}{|c|}{ 2. Preliminary design-design calculations based on initial material } \\
\hline \multicolumn{3}{|c|}{ 3. Design for additive manufacturing } \\
\hline $\begin{array}{l}\text { Build time, support } \\
\text { structure, quality, DFA, } \\
\text { build orientation }\end{array}$ & $\begin{array}{l}\text { Material properties, } \\
\text { structural integrity }\end{array}$ & $\begin{array}{l}\text { AM Process and its } \\
\text { parameters }\end{array}$ \\
\hline \multicolumn{3}{|c|}{ 4. Material mesostructure modeling and AM process modeling } \\
\hline \multicolumn{3}{|c|}{ 5. Analyze design } \\
\hline \multicolumn{3}{|c|}{ 6. Redesign with actual material properties } \\
\hline \multicolumn{3}{|c|}{ 7. Optimize } \\
\hline & 8. 3D print & \\
\hline
\end{tabular}

\section{CONCLUSIONS}

The additive manufacturing technologies facilitated more freedom for fabrication of any shape of the part and with additional benefits such as rapid prototyping, no tooling and minimal or no wastage of material. However, the changes in the material properties of a part due to the physical phenomena that is taking places during layer upon layer deposition of a material need to be addressed. The change in the material behavior greatly influences the design and analysis of the parts for 3D printing. The anisotropy and inhomogeneity in the material properties do not allow the designer for effective design of the parts. Also, designing of a part for 3D printing requires to consider influence of other factors that include type of AM process and its parameters and build orientation on build time, cost, support structure 
and surface quality of the parts. Further, the relation between the process-structure-property-performance is need to be considered during the design stage of a part for 3D printing for effective design and analysis.

\section{REFERENCES}

[1] Standard Terminology for Additive Manufacturing Technologies 1,2, (n.d.). doi:10.1520/F2792-12A.

[2] G. A. Adam, and D. Zimmer, "On design for additive manufacturing: evaluating geometrical limitations". Rapid Prototyping Journal, vol. 21(6), pp. 662-670, 2015.

[3] C. Mueller, A. Irani and B. Jenett, "Additive manufacturing of structural prototypes for conceptual design", In Proceedings of the IASS-SLTE Symposium, Shells, Membranes and Spatial Structures: Footprints, Brasil and Pauletti (Eds.), Brasilia, Brazil 2014 Sep 15.
[4] D. W. Rosen, "Research supporting principles for design for additive manufacturing: This paper provides a comprehensive review on current design principles and strategies for AM". Virtual and Physical Prototyping, vol. 9(4), pp.225-232, 2014.

[5] J. Kotlinski, "Mechanical properties of commercial rapid prototyping materials", Rapid Prototyping Journal, vol. 20(6), pp.499-510, 2014.

[6] A. Bagsik, V. Schöppner, and E. Klemp, "FDM part quality manufactured with Ultem* 9085", In 14th international scientific conference on polymeric materials, Vol. 15, pp. 307-315, 2010.

[7] R. Hague, S. Mansour, N. Saleh, and R. Harris, "Materials analysis of stereolithography resins for use in rapid manufacturing", Journal of materials science, vol. 39(7), pp.2457-2464, 2004.

[8] D. Agius, K.I. Kourousis, and C. Wallbrink, "A Review of the As-Built SLM Ti-6Al-4V Mechanical Properties towards Achieving Fatigue Resistant Designs". Metals, 8(1), p.75, 2018. 